\title{
Algebraic growth in a Blasius boundary layer: Nonlinear optimal disturbances
}

\author{
Simone Zuccher ${ }^{\mathrm{a}}$, Alessandro Bottaro ${ }^{\mathrm{b}}$, Paolo Luchini ${ }^{\mathrm{c}, *}$ \\ ${ }^{a}$ Aerospace and Mechanical Engineering Department, The University of Arizona, 1130 N. Mountain Ave., Tucson, AZ 85721, USA \\ b DIAM, Università di Genova, Via Montallegro 1, 16145 Genova, Italy \\ c Dipartimento di Ingegneria Meccanica, Università di Salerno, 84084 Fisciano, Italy
}

Received 30 June 2004; received in revised form 7 April 2005; accepted 9 July 2005

Available online 1 September 2005

\begin{abstract}
The three-dimensional, algebraically growing instability of a Blasius boundary layer is studied in the nonlinear regime, employing a nonparallel model based on boundary layer scalings. Adjoint-based optimization is used to determine the "optimal" steady leading-edge excitation that provides the maximum energy growth for a given initial energy. Like in the linear case, the largest transient growth is found for inlet streamwise vortices, that yield streamwise streaks downstream. Two different definitions of growth are employed, providing qualitatively similar results, although the spanwise wavenumbers of optimal growth differ by up to $20 \%$ in the two cases. The wavelength of the most amplified optimal disturbance increases with the initial amplitude. For large input amplitudes, significant deformations of the mean velocity field are found; in such cases it is reasonable to expect that nonlinear streaks may break down through a secondary instability.
\end{abstract}

() 2005 Elsevier SAS. All rights reserved.

Keywords: Boundary layer; Stability; Transient growth; Nonlinear; Optimal perturbation

\section{Introduction}

\subsection{Algebraic instability}

Classical eigenvalue-based linear stability theory sometimes predicts stable behaviour for flows which experiments show to become turbulent. For instance, the stability analysis of Hagen-Poiseuille pipe flow reveals that all eigenfunctions are stable; however, if the Reynolds number $R e$ is large enough, transition is observed. In particular, recent experimental studies by Hof et al. [1] have shown that the finite amplitude of the disturbance necessary to trigger transition in a pipe flow scales as $\mathcal{O}\left(R e^{-1}\right)$ beyond a certain $R e$-threshold. Moreover for certain flows, for example plane Poiseuille flow, transition practically occurs at a Reynolds number considerably smaller than the critical value provided by the theory. This indicates that there must exist a mechanism for the growth of disturbances which does not rely on the fact that (at least) one eigenmode of the Orr-Sommerfeld equation has positive amplification factor.

\footnotetext{
* Corresponding author.

E-mail addresses: zuccher@email.arizona.edu (S. Zuccher), bottaro@diam.unige.it (A. Bottaro), luchini@unisa.it (P. Luchini).
} 
Ellingsen and Palm [2] and Landahl [3] identified an alternative mechanism for the growth of perturbations and described a new phenomenon in wall-bounded flows according to which a longitudinal vortex superimposed to a two-dimensional boundary layer can lift up low-velocity fluid from the wall and push down high-velocity fluid towards the wall. ${ }^{1}$ Since the structure of the boundary layer is elongated in the streamwise direction, with a typical length $R e^{1 / 2}$ times greater than the boundary layer thickness, the disturbance accumulated over the streamwise length can be $R e^{1 / 2}$ times greater than the original one.

The combination of this basically inviscid amplification mechanism with the damping effect of viscosity leads to what is nowadays called algebraic instability or transient growth. This mechanism is also believed to be at the origin of the experimentally observed pattern known as Klebanoff modes which appear to be the precursor of the so-called bypass transition (Morkovin [5] and Morkovin and Reshotko [6]). Recent studies [7] show that in a spatially growing boundary layer, as opposed to parallel pipe or channel flow, viscosity is not sufficient to damp the algebraic growth for streaks of sufficiently large spanwise wavelength, so that the receptivity to low-amplitude streamwise vortices can play an important role in boundary layer transition.

\subsection{Previous work on algebraic growth}

Receptivity to upstream disturbances which can lead to boundary-layer transition via the transient growth mechanism was first investigated by seeking the initial conditions, for the boundary layer past a flat plate, that produced the largest energy amplification. This is known as the "optimal perturbation" approach.

All previously published work in this field is limited to a linearized analysis. Insofar as the temporal stability problem is concerned, optimal perturbations were first so named by Farrell [8] to denote the initial flow disturbances that produced the maximum gain, defined as the ratio between the perturbation kinetic energies at the final and initial time. This first approach related to a two-dimensional plane channel flow. Actually, Boberg and Brosa [9] had already introduced a similar concept for flow in a pipe, but Butler and Farrell [10] gave the first quantitative calculation of three-dimensional optimal perturbations with respect to temporal growth, not only for plane Couette and Poiseuille flow, but also for a parallel approximation of the Blasius boundary layer. Corbett and Bottaro [11] employed an optimal-control type of analysis to find the temporally growing optimal perturbations for parallel boundary layers subject to streamwise pressure gradient; later, they studied the temporal growth in swept boundary layers described by Falkner-Skan-Cooke similarity solution [12].

The problem of spatial stability was recently tackled by Luchini $[13,14]$ and Andersson et al. $[15,16]$. They found that the optimal initial disturbance is composed of stationary streamwise vortices whereas the induced velocity field is dominated by streamwise streaks. The maximum amplification occurs in the steady case (frequency $\omega=0$ ) and for a non-zero value of the spanwise wavenumber $\beta=0.45$ (scaled with $\delta=\sqrt{v L / U_{\infty}}, L$ being the longitudinal distance from the leading edge to the location where output energy is maximized).

Andersson et al. [17], in the effort to identify a path of bypass transition, investigated via direct numerical simulation the subsequent nonlinear evolution of the optimal perturbations given by the linear approach (those computed by Luchini [14] and Andersson et al. [16]), focusing upon the "secondary" temporal instability of the streaks produced by the algebraic mechanism. Results show that the streak's critical amplitude, beyond which streamwise travelling waves are excited, is about $26 \%$ of the free-stream velocity. They did not actually optimize the perturbations in the nonlinear case. Experimental evidence of the growth of streaks and their breakdown is provided by Matsubara and Alfredsson [18]. Their study furnishes encouraging data for the theories of algebraic growth and secondary instability and, at the same time, it indicates that large amplitude effects can be the cause of a quantitative mismatch between the predicted spanwise wavenumber of the streaky structures and the measured one.

A different approach to the study of Klebanoff modes was pursued by Wundrow and Goldstein [19]. Rather than searching for the optimal initial disturbance profile, they considered the effect of a small-amplitude, steady streamwise-vorticity field superposed to the flow over an infinitely thin flat plate. By breaking the domain into several regions, described by different asymptotic approximations, Wundrow and Goldstein demonstrated that nonlinear, localized shear layers are produced downstream of the leading edge, with qualitative similarities with the direct numerical simulation results of Jacobs and Durbin [20] of a boundary layer subject to free-stream turbulence. Unlike the present work, the study by Wundrow and Goldstein did not involve any optimization; still, it paved important new ground for the understanding of the breakdown of streaks, and the ensuing bypass transition phenomenon.

\subsection{Emerging theories of transition}

Transition to turbulence is a phenomenon dominated by nonlinear effects; it is thus important to briefly describe some very recent developments that appear very promising in the pursuit of a satisfactory description of transition. The so-called theory of

\footnotetext{
1 A similar amplification mechanism based on the interaction of shear with longitudinal vortices had even earlier been proposed by Moffatt [4] as the generation mechanism of large-scale structures in self-sustained free-shear turbulence and the source of elastic behaviour in its average stress-strain relationship.
} 
the self-sustained process [21-23] arose out of efforts to understand how transitional and turbulent states are maintained near walls by nonlinear feedback processes. Schematically such a process starts with the formation of longitudinal streaks out of streamwise vortices through the lift-up effect, the breakdown of the streaks with the creation of travelling waves, and the vortex regeneration process. Whereas each individual step of the process can be isolated and studied per se (cf. the work by Andersson et al. [17] on the breakdown of streaks, for example) only the consideration of finite amplitude states consisting simultaneously of streaks, rolls and travelling waves provides the mean to each individual component of the process to sustain one another against viscous decay. Once the realization came about that finite amplitude equilibrium solutions had to be searched for, containing all of the "ingredients" above, the study of so-called "exact coherent structures" started. Without dwelling into too many details, out of the scope of the present paper, we limit ourselves in pointing out that such a search has already produced a remarkable success story for the case of the turbulent pipe flow: experimental results [24] and theory [25,26] provide flow structures in such close agreement to one another to cause an outflow of papers in the popular science press (consult, for example, the article by F. Busse, "Visualizing the dynamics of the onset of turbulence", in the September 10, 2004 issue of Science, or that by C. Barenghi, "Turbulent transition for fluids", in the December 2004 issue of Physics World, or that in the Novembre 2004, News Scan Briefs section of Scientific American, by the title "Piped-in Turbulence").

\subsection{Goal of the present work}

After having pointed out the importance of nonlinearities, we feel that there is ample justification in developing a numerical optimization procedure to search for nonlinear optimal disturbances by solving the complete boundary-layer equations.

Limiting the study to the linear approach yields a result which is independent of the initial energy of the perturbation, since the whole disturbance field is defined up to a multiplicative constant. In the nonlinear evolution, once the energy has grown beyond a certain threshold, nonlinear interactions begin to compensate for the amplification, producing a state which eventually saturates. In this work we will limit ourselves to presenting the procedure and to optimizing the state with respect to the disturbance kinetic energy of the flow, validating against known linear solutions. It could then be possible (and in fact it is quite easy) to extend the present procedure to optimize other functionals, arguably more relevant in the context of transition, such as the rate of disturbance energy production or dissipation (cf. the recent work by Plasting and Kerswell [27]), providing a link to the emerging theories of transition briefly outlined in the previous section.

\section{Problem formulation}

The algebraic instability in the Blasius boundary layer is here studied in the context of the steady three-dimensional incompressible boundary-layer equations. They are simpler to handle than the more general Navier-Stokes equations in that, since they are parabolic in the streamwise direction, a marching technique is allowed for their numerical solution. In addition they contain one dimensionless parameter less and provide a Reynolds-number-independent result. However, one must consider that two different three-dimensional formulations of the boundary layer approximation exist, depending on whether the spanwise scale of the phenomenon considered is comparable to the streamwise scale $L$ or to the normal scale (as pointed out, among others, by Luchini [7]). Both are consistent inner-outer expansions of the complete problem, but for different scalings of the spanwise coordinate and velocity. The first case is typical of a three-dimensional outer stream, while the second is typical of three-dimensionalities originating inside the boundary layer itself, such as, for example, Görtler vortices. For this reason we use the second formulation. The equations, written in boundary-layer variables and in conservative form, read:

$$
\begin{aligned}
& u_{x}+v_{y}+w_{z}=0, \\
& (u u)_{x}+(u v)_{y}+(u w)_{z}-u_{y y}-u_{z z}=0, \\
& (u v)_{x}+(v v)_{y}+(v w)_{z}+p_{y}-v_{y y}-v_{z z}=0, \\
& (u w)_{x}+(v w)_{y}+(w w)_{z}+p_{z}-w_{y y}-w_{z z}=0,
\end{aligned}
$$

where the streamwise velocity component $u$ is made dimensionless with respect to the outer velocity $U_{\infty}$, whereas $v$ and $w$ (respectively wall-normal and spanwise components) are made dimensionless with respect to $\operatorname{Re}-1 / 2 U_{\infty}$. Re is the Reynolds number defined as $R e=U_{\infty} L / v$. The streamwise coordinate $x$ is normalized with a reference length $L$, the wall-normal coordinate $y$ and the spanwise coordinate $z$ are made dimensionless with $\delta=R e^{-1 / 2} L=\left(v L / U_{\infty}\right)^{1 / 2}$. Pressure is normalized with $R e^{-1} \rho U_{\infty}^{2}, \rho$ being the density. It should be emphasized that what we call $p$ here is the second-order term in the usual 
inner expansion of pressure, both the zero- and first-order terms being zero for Blasius flow. System (1) requires six boundary conditions, three at the wall and three in the free stream:

$$
\begin{array}{llll}
u=0 & \text { at } y=0, & u=1 & \text { for } y \rightarrow \infty, \\
v=0 & \text { at } y=0, & w=0 & \text { for } y \rightarrow \infty, \\
w=0 & \text { at } y=0, & p=0 & \text { for } y \rightarrow \infty .
\end{array}
$$

The system is parabolic in $x$ and therefore initial conditions are also required. However, only two initial conditions are allowed for the boundary-layer equations, as opposed to three for the Navier-Stokes equations, so that if $u$ is fixed, $v$ and $w$ must be related to each other. This can be proved [28,29] by combining the continuity equation and the $x$-momentum equation so as to eliminate $u_{x}$ between them, which gives an equation without any $x$-derivative nor pressure term. The latter represents a constraint to be satisfied locally at any $x$, including $x=0$ : of the three initial velocity components only two are thus independent. Moreover, when the streamwise component $u$, upstream of the leading edge, equals 1 uniformly in $x, y$ and $z$, the constraint binding the initial conditions simply reduces to the continuity equation. The initial conditions we shall consider therefore are

$$
\begin{aligned}
& u(0, y, z)=1, \\
& v(0, y, z)=v_{0}(y, z),
\end{aligned}
$$

$w(0, y, z)=w_{0}(y, z)$ being consequently derived from $v_{0}$ and the constraint $w_{z}=-v_{y}$.

System (1) with initial conditions (3) and boundary conditions (2) represents the "direct" (or "forward") problem to be solved. The next step in the formulation consists in identifying an objective function physically related to the stability characteristics of the flow, so that its maximization yields the worst possible scenario.

\subsection{Choice of the objective function}

In previous work on optimal perturbations, the perturbation kinetic energy was typically chosen as a measure of the disturbance. In lack of a better "harmfulness" estimator, this seems to be a reasonable physical quantity. In our problem, however, since a complete nonlinear calculation is performed, the flow field is not just decomposed as a base flow plus a small perturbation, but as a sum of Fourier modes in the spanwise direction (cf. Appendix A). Mode zero contains both the unperturbed flow (Blasius' solution as we are dealing with a flat plate) and a mean-flow correction due to the interactions of the other modes. Different energy definitions are therefore possible. The integral along $y$ and $z$ of the kinetic energy of all modes, including mode zero, is not a good choice because it is infinite (both $u$ and $v$ tend to a constant as $y \rightarrow \infty$ ). This could be avoided by taking, instead of mode zero, only the mean flow correction, i.e. the difference between the total velocity field and Blasius' solution. This is not the typical choice of experimentalists (cf. Matsubara and Alfredsson [18]), who cannot easily distinguish between base flow and mean flow correction, once nonlinearities kick in. Hence, as disturbance measure we have chosen the kinetic energy of the oscillating part of the velocity, i.e. the sum of the energies of all modes, excluding mode zero. When integrated along $y$ and $z$, this energy is a function of the streamwise coordinate only and its evolution from the inlet to the outlet is a good indicator of the perturbation growth.

Once physical dimensions are taken into account, because of the different scaling of streamwise and crossflow velocity components in the boundary-layer formulation, the perturbation kinetic energy as a function of $x$ contains $R e$ and reads:

$$
E(x)=\frac{1}{2 Z} \int_{-Z}^{Z} \int_{0}^{\infty}\left[\left|u^{\prime}\right|^{2}+R e^{-1}\left(\left|v^{\prime}\right|^{2}+\left|w^{\prime}\right|^{2}\right)\right] \mathrm{d} y \mathrm{~d} z,
$$

with primes denoting the oscillating part of each variable with mode zero excluded, and $Z=\pi / \beta$. In an input-output framework, however, the interest is typically in the ratio between the output energy and the input energy, as it represents the gain of the system. This can have at least two expressions. The first is the one used by Luchini [14] and Andersson et al. [16] for the optimal-perturbation problem:

$$
G_{\text {out }}=\frac{E_{\text {out }}}{E_{\text {in }}}=\frac{\left[(1 /(2 Z)) \int_{-Z}^{Z} \int_{0}^{\infty}\left[\left|u^{\prime}\right|^{2}+R e^{-1}\left(\left|v^{\prime}\right|^{2}+\left|w^{\prime}\right|^{2}\right)\right] \mathrm{d} y \mathrm{~d} z\right]_{x=1}}{\left[(1 /(2 Z)) \int_{-Z}^{Z} \int_{0}^{\infty}\left[\left|u^{\prime}\right|^{2}+R e^{-1}\left(\left|v^{\prime}\right|^{2}+\left|w^{\prime}\right|^{2}\right)\right] \mathrm{d} y \mathrm{~d} z\right]_{x=0}} .
$$

A second possible one is given below:

$$
G_{\text {mean }}=\frac{E_{\text {mean }}}{E_{\text {in }}}=\frac{(1 /(2 Z)) \int_{-Z}^{Z} \int_{0}^{\infty} \int_{0}^{1}\left[\left|u^{\prime}\right|^{2}+R e^{-1}\left(\left|v^{\prime}\right|^{2}+\left|w^{\prime}\right|^{2}\right)\right] \mathrm{d} x \mathrm{~d} y \mathrm{~d} z}{\left[(1 /(2 Z)) \int_{-Z}^{Z} \int_{0}^{\infty}\left[\left|u^{\prime}\right|^{2}+R e^{-1}\left(\left|v^{\prime}\right|^{2}+\left|w^{\prime}\right|^{2}\right)\right] \mathrm{d} y \mathrm{~d} z\right]_{x=0}} .
$$


Expression (6) was used by Cathalifaud and Luchini [30] for their optimal-control problem, as they found that optimal control based on minimizing the final energy (5) can produce very large overshoots in the disturbance energy curve at intermediate positions before the end station, with obvious undesirable consequences on boundary layer transition.

Since the aim is to maximize $G$, by looking at the denominators of expressions (5) and (6) the obvious best choice is $u^{\prime}(0, y, z)=0$. This yields a $G$ which is $\mathcal{O}(R e)$ when $R e \rightarrow \infty$, whereas $u^{\prime}(0, y, z) \neq 0$ would lead to $G$ of order $\mathcal{O}(1)$. The term $R e^{-1}\left(\left|v^{\prime}\right|^{2}+\left|w^{\prime}\right|^{2}\right)$ in the numerators, as noted by Luchini [14], is inconsistent with the inner-outer expansion from which the boundary-layer approximation is derived, unless higher-order terms are included in the inner-outer expansion itself. By introducing the new definitions

$$
E_{u}(x)=\frac{1}{2 Z} \int_{-Z}^{Z} \int_{0}^{\infty}\left|u^{\prime}\right|^{2} \mathrm{~d} y \mathrm{~d} z ; \quad E_{\text {in }}=\left[\frac{1}{2 Z} \int_{-Z}^{Z} \int_{0}^{\infty}\left[\left|v^{\prime}\right|^{2}+\left|w^{\prime}\right|^{2}\right] \mathrm{d} y \mathrm{~d} z\right]_{x=0},
$$

expressions (5) and (6) reduce at leading order to:

$$
G_{\text {out }}=\operatorname{Re} \frac{E_{u}(1)}{E_{\text {in }}} ; \quad G_{\text {mean }}=\operatorname{Re} \frac{\int_{0}^{1} E_{u}(x) \mathrm{d} x}{E_{\text {in }}},
$$

and contain the Reynolds number $R e$ as a scaling factor only. Thus, also in the nonlinear regime the gain of whatever optimal perturbation we shall find scales linearly with the Reynolds number.

In order to evaluate the pertinence of the two objective functions defined in (8), we introduce the weighted sum

$$
\mathcal{J}=\alpha_{1} E_{u}(1)+\alpha_{2} \int_{0}^{1} E_{u}(x) \mathrm{d} x,
$$

also noted as $\mathcal{J}=\alpha_{1} E_{\text {out }}+\alpha_{2} E_{\text {mean }}$, so that for the special case $\alpha_{1}=1$ and $\alpha_{2}=0$ the objective is the maximization of the energy at the final station, whereas for $\alpha_{1}=0$ and $\alpha_{2}=1$ the aim is to maximize the integral of the energy over the whole domain.

\subsection{Constrained optimization and discrete adjoint problem}

The problem of finding the leading-edge perturbation that optimizes a given cost function $\mathcal{J}$ simply translates in a constrained optimization where the constraints are $E_{\mathrm{in}}\left(v_{0}, w_{0}\right)=E_{0}$ and the governing equations (1) with associated boundary and initial conditions (2), (3). As stated earlier, since $w_{0}$ is related to $v_{0}$, the problem reduces to finding $v_{0}$.

A classical tool for the solution of constrained optimization problems is the Lagrange multiplier technique. It consists in first introducing the augmented functional $\mathcal{L}$, which contains the cost $\mathcal{J}$, the constraints and the Lagrange multipliers. Since the constraint (1) is in a differential form, integration by parts over the whole domain is required. Then, by imposing $\delta \mathcal{L}=0$, i.e. that the variation of $\mathcal{L}$ with respect to each of its independent variables must be zero, a set of adjoint equations along with their boundary conditions (at $y=0$ and $y \rightarrow \infty$ ) and "initial" conditions (at $x=X$ ) are obtained. Moreover, the procedure furnishes the optimality condition at $x=0$, which provides the expression of $v_{0}$ as a function of the adjoint field. The details of the derivation of the adjoint equations in the continuous framework have already been given by Zuccher et al. [31]. The set of linear partial differential equations so obtained can be discretized and solved numerically by writing a new code from scratch, possibly with a methodology inspired to that employed for the direct problem (Appendix A).

On the other hand, it is also possible to solve the constrained optimization by applying the classical Lagrange multiplier technique to the discrete direct problem. In doing so, the need of an "ad hoc" adjoint code is avoided and, in the context of optimization, it is not even necessary to give a continuous interpretation to the discrete adjoint equations; it suffices to know that adjoint-based optimization of the discrete equations will give an exact optimum of the discrete problem for any step size. Provided the discrete problem is a consistent approximation of the continuous direct problem for step size tending to zero, and provided the optimum is a-posteriori checked to lie within the range of initial conditions for which the approximation of the direct problem converges, the correct result will be obtained. For further examples the reader is referred to Luchini and Bottaro [29,32], Luchini [14] and Cathalifaud and Luchini [30]. For a thorough discussion on the issue of continuous versus discrete adjoint we refer to Gunzburger [33]; for examples of inconsistent direct/adjoint discretization the reader can consult Moore and Farrell [34].

The numerical discretization of a general parabolic system of partial differential equations such as (1) can be recast as

$$
\mathbf{A}_{n+1} \mathbf{f}_{n+1}=\mathbf{B}_{n} \mathbf{f}_{n}
$$

where $n$ denotes the $n$-th grid point in the streamwise direction $x, \mathbf{f}$ is the vector of unknowns and matrices $\mathbf{A}$ and $\mathbf{B}$ depend on $x$ (as the base flow does) and account for the discretization in $x, y$ and $z$ (in our case respectively second-order differences 
on uneven grids for both $x$ and $y$ and Fourier decomposition in $z$ ). The solution is found by marching forward in space from $n=0$, given the initial condition $\mathbf{f}_{0}$, to $n=N-1$. The boundary conditions at the wall and for $y \rightarrow \infty$ are already included in matrices' rows. The energy norm of the initial perturbation, in the discrete setting, can be expressed as

$$
E_{\text {in }}=\mathbf{f}_{0}^{\mathrm{T}} \mathbf{M}_{0} \mathbf{f}_{0}=E_{0}
$$

and the objective function as $\mathcal{J}=\alpha_{1} \mathbf{f}_{N}^{\mathrm{T}} \mathbf{M}_{N} \mathbf{f}_{N}+\alpha_{2} \sum_{n=1}^{N} \mathbf{f}_{n}^{\mathrm{T}} \mathbf{M}_{n} \mathbf{f}_{n}$ (matrices $\mathbf{M}_{0}, \mathbf{M}_{n}$ and $\mathbf{M}_{N}$ contain, again, the discretization in all directions). The application of Lagrange multipliers to the discretized equations involves the same conceptual steps as its continuous counterpart. The augmented functional $\mathcal{L}$, which contains the cost $\mathcal{J}$, the constraints (9) and (10), and the Lagrange multipliers is written as

$$
\mathcal{L}\left(\mathbf{f}_{0}, \mathbf{f}_{n}, \mathbf{f}_{n+1}, \mathbf{f}_{N}\right)=\alpha_{1} \mathbf{f}_{N}^{\mathrm{T}} \mathbf{M}_{N} \mathbf{f}_{N}+\alpha_{2} \sum_{n=1}^{N} \mathbf{f}_{n}^{\mathrm{T}} \mathbf{M}_{n} \mathbf{f}_{n}+\sum_{n=0}^{N-1}\left[\mathbf{p}_{n}^{\mathrm{T}}\left(\mathbf{A}_{n+1} \mathbf{f}_{n+1}-\mathbf{B}_{n} \mathbf{f}_{n}\right)\right]+\lambda_{0}\left[\mathbf{f}_{0}^{\mathrm{T}} \mathbf{M}_{0} \mathbf{f}_{0}-E_{0}\right]
$$

where $\mathbf{p}_{n}$ is the vector of Lagrange multipliers, function of the streamwise location $n$. Only the dependence on $\mathbf{f}$ has been emphasized in $\mathcal{L}$ since derivation of $\mathcal{L}$ with respect to the Lagrange multipliers (required to enforce $\delta \mathcal{L}=0$ ) would simply reproduce Eq. (9). The summation between 0 and $N-1$ involving $\mathbf{p}_{n}$ reflects the integral along $x$. The integration by parts is here replaced by adding and subtracting $\mathbf{p}_{n+1}^{\mathrm{T}} \mathbf{B}_{n+1} \mathbf{f}_{n+1}$ so that the terms can be rearranged as

$$
\begin{aligned}
\sum_{n=0}^{N-1}\left[\mathbf{p}_{n}^{\mathrm{T}}\left(\mathbf{A}_{n+1} \mathbf{f}_{n+1}-\mathbf{B}_{n} \mathbf{f}_{n}\right)\right] & =\sum_{n=0}^{N-1}\left[\mathbf{p}_{n}^{\mathrm{T}} \mathbf{A}_{n+1} \mathbf{f}_{n+1}-\mathbf{p}_{n+1}^{\mathrm{T}} \mathbf{B}_{n+1} \mathbf{f}_{n+1}\right]+\sum_{n=0}^{N-1}\left[\mathbf{p}_{n+1}^{\mathrm{T}} \mathbf{B}_{n+1} \mathbf{f}_{n+1}-\mathbf{p}_{n}^{\mathrm{T}} \mathbf{B}_{n} \mathbf{f}_{n}\right] \\
& =\sum_{n=0}^{N-1}\left[\mathbf{p}_{n}^{\mathrm{T}} \mathbf{A}_{n+1} \mathbf{f}_{n+1}-\mathbf{p}_{n+1}^{\mathrm{T}} \mathbf{B}_{n+1} \mathbf{f}_{n+1}\right]+\mathbf{p}_{N}^{\mathrm{T}} \mathbf{B}_{N} \mathbf{f}_{N}-\mathbf{p}_{0}^{\mathrm{T}} \mathbf{B}_{0} \mathbf{f}_{0},
\end{aligned}
$$

and expression (11) can be rewritten as

$$
\begin{aligned}
\mathcal{L}\left(\mathbf{f}_{0}, \mathbf{f}_{n+1}, \mathbf{f}_{N}\right)= & \alpha_{1} \mathbf{f}_{N}^{\mathrm{T}} \mathbf{M}_{N} \mathbf{f}_{N}+\alpha_{2} \sum_{n=0}^{N-1} \mathbf{f}_{n+1}^{\mathrm{T}} \mathbf{M}_{n+1} \mathbf{f}_{n+1}+\sum_{n=0}^{N-1}\left[\mathbf{p}_{n}^{\mathrm{T}} \mathbf{A}_{n+1} \mathbf{f}_{n+1}-\mathbf{p}_{n+1}^{\mathrm{T}} \mathbf{B}_{n+1} \mathbf{f}_{n+1}\right] \\
& +\mathbf{p}_{N}^{\mathrm{T}} \mathbf{B}_{N} \mathbf{f}_{N}-\mathbf{p}_{0}^{\mathrm{T}} \mathbf{B}_{0} \mathbf{f}_{0}+\lambda_{0}\left[\mathbf{f}_{0}^{\mathrm{T}} \mathbf{M}_{0} \mathbf{f}_{0}-E_{0}\right] .
\end{aligned}
$$

It should be noticed that the summation with $\alpha_{2}$ as a pre-factor covers the range from 0 to $N-1$ (instead of 1 to $N$ ) in order to drop the dependence on $\mathbf{f}_{n}$ in $\mathcal{L}$.

As in the continuous case, the extremum condition is found when $\delta \mathcal{L}=0$, i.e.

$$
\frac{\mathcal{D} \mathcal{L}}{\mathcal{D} \mathbf{f}_{0}} \delta \mathbf{f}_{0}+\sum_{n=0}^{N-2} \frac{\mathcal{D} \mathcal{L}}{\mathcal{D} \mathbf{f}_{n+1}} \delta \mathbf{f}_{n+1}+\frac{\mathcal{D} \mathcal{L}}{\mathcal{D} \mathbf{f}_{N}} \delta \mathbf{f}_{N}=0,
$$

which, in order to be satisfied for any arbitrary $\mathbf{f}_{0}, \mathbf{f}_{n+1}$ and $\mathbf{f}_{N}$, leads to

$$
\begin{aligned}
& \frac{\mathcal{D} \mathcal{L}}{\mathcal{D} \mathbf{f}_{0}}=-\mathbf{p}_{0}^{\mathrm{T}} \mathbf{B}_{0}+2 \lambda_{0} \mathbf{f}_{0}^{\mathrm{T}} \mathbf{M}_{0}=0, \\
& \sum_{n=0}^{N-2} \frac{\mathcal{D} \mathcal{L}}{\mathcal{D} \mathbf{f}_{n+1}}=\sum_{n=0}^{N-2}\left[\mathbf{p}_{n}^{\mathrm{T}} \mathbf{A}_{n+1}-\mathbf{p}_{n+1}^{\mathrm{T}} \mathbf{B}_{n+1}+2 \alpha_{2} \mathbf{f}_{n+1}^{\mathrm{T}} \mathbf{M}_{n+1}\right]=0, \\
& \frac{\mathcal{D} \mathcal{L}}{\mathcal{D} \mathbf{f}_{N}}=2 \alpha_{1} \mathbf{f}_{N}^{\mathrm{T}} \mathbf{M}_{N}+\mathbf{p}_{N}^{\mathrm{T}} \mathbf{B}_{N}=0 .
\end{aligned}
$$

Eq. (13) furnishes the optimality condition to be satisfied at $x=0$ and Eq. (14) leads to

$$
\mathbf{A}_{n+1}^{\mathrm{T}} \mathbf{p}_{n}=\mathbf{B}_{n+1}^{\mathrm{T}} \mathbf{p}_{n+1}-2 \alpha_{2} \mathbf{M}_{n+1}^{\mathrm{T}} \mathbf{f}_{n+1},
$$

which is nothing but the discrete form of the adjoint equations to be solved by marching backwards from $x=X$ to $x=0$ with the initial condition derived from Eq. (15) solved for $\mathbf{p}_{N}$. 


\subsection{Iterative optimization}

The equations and conditions derived in the previous sections for the direct and adjoint problems can in principle be satisfied by solving the complete forward and backward problem as one big coupled system. However, doing so would require a considerable computational effort and would not put the parabolic character of the equations to good use.

On the contrary, when seen separately the equations are parabolic in either the forward (direct problem) or backward (adjoint problem) direction, suggesting the idea of an iterative approach. This involves alternating the solution of the direct and adjoint equations until a converged solution is hopefully attained. At this point $\delta \mathcal{L}=0$ will have been achieved. The procedure can be summarized as follows:

1. An initial guess is taken for the initial condition $\mathbf{f}_{0}$ at $x=0$.

2. The parabolic direct problem (9), which is the discretized version of (1), is solved by marching forward in space from $x=0$ to $x=1(n=0, \ldots, N-1)$.

3. At $x=1(n=N)$ Eq. (15) solved for $\mathbf{p}_{N}$ provides the initial condition for the adjoint solution. If the objective is the maximization of the integral of the energy over the whole domain (i.e. if $\alpha_{1}=0$ ), the condition is simply $\mathbf{p}_{N}=0$.

4. The adjoint problem (16) is marched backward in space from $x=1$ to $x=0(n=N-1, \ldots, 0)$, starting from the initial condition obtained at step 3 . If the objective includes the mean energy, i.e. $\alpha_{2} \neq 0$, in the equations there is a forcing term containing the (now known) direct solution $2 \alpha_{2} \mathbf{M}_{n+1}^{\mathrm{T}} \mathbf{f}_{n+1}$.

5. Once the adjoint solution is found, the optimality condition (13) determines the new initial condition for the direct problem $\mathbf{f}_{0}$ and the Lagrange multiplier $\lambda_{0}$ is chosen so that the constraint $E_{\mathrm{in}}\left(\mathbf{f}_{0}\right)=E_{0}$ is satisfied. Since in the nonlinear case convergence of a simple iteration cannot be assured, a relaxation involving the value of $\mathbf{f}_{0}$ at the previous iteration can be introduced if necessary.

6. Step 2 is repeated using the initial conditions updated at step 5. The objective function $\mathcal{J}$ is evaluated again and compared with its previous value: if a smaller $\mathcal{J}$ results, the relaxation parameter employed in the gradient method is halved and step 2 repeated until an increase of $\mathcal{J}$ appears (this is equivalent to a successive-bisection search, as explained in the next paragraph).

The complete procedure is repeated until the difference in $\mathcal{J}$ between two successive iterations is smaller than a given threshold. This is similar to the algorithm used, for the corresponding linear problem, by Luchini [14]. In that case, the iterative optimization technique reduced to performing power iterations for the maximization of a Rayleigh quotient, a procedure which is mathematically guaranteed to converge. Here the problem is nonlinear, and the optimization does not reduce to a Rayleigh quotient; convergence cannot be mathematically assured and occasionally may not occur for very large values of the initial energy. However, since a well defined objective function can be computed at every step, convergence can easily be restored by a successive-bisection search, applied at the point in the loop where the coupling condition is imposed at $x=0$. This means that, instead of applying condition (13) straightforwardly, we introduce a parameter $k \in[0,1]$ such that the new initial condition is updated partly with its value at the previous forward-backward iteration $(i)$ and partly with the solution coming from the adjoint field at the same forward-backward iteration $(i)$ :

$$
\mathbf{f}_{0}^{(i+1)}=(1-k) \mathbf{f}_{0}^{(i)}+\frac{k}{2 \lambda_{0}^{(i)}}\left[\mathbf{M}_{0}^{\mathrm{T}}\right]^{-1} \mathbf{B}_{0}^{\mathrm{T}} \mathbf{p}_{0}^{(i)} .
$$

Condition (13) is recovered for $k=1$. In practice, after completing steps 1 to 5 above, step 2 is repeated and the objective function calculated and compared with its previous value. If a reduction is observed rather than an increase, the result is discarded and step 2 repeated with a halved value of $k$, until a monotonic increase in the objective function is achieved. This bisection-search procedure was needed only for the highest considered value of the initial disturbance energy $\left(E_{0}=500\right)$. In that case the increment was halved 3 times at about the third or fourth forward iteration, depending on the wavenumber $\beta$. In all other cases, the relaxation factor $k$ was never halved.

\section{Parametric study}

For the reasons outlined in Section 2.1, at $x=0 u_{0}$ is fixed and equal to 1 , while $w_{0}$ can be derived from $v_{0}$, which is the only unknown. In Fourier space, the set of initial conditions is:

$$
\begin{array}{ll}
\text { mode } n=0 & \text { mode } n \geqslant 1 \\
U_{0}(0, y)=1, & U_{n}(0, y)=0, \\
V_{0}(0, y)=0, & V_{n}(0, y)=V_{n}^{0}(y), \\
W_{0}(0, y)=0, & W_{n}(0, y)=W_{n}^{0}(y) .
\end{array}
$$


Results shown below have been verified for grid convergence by repeating the computation over more refined grids. Numerical tests indicate that an adequate number of grid points is 200 along $x$ (clustered near the leading edge) and 300 along $y$ (clustered near the wall). The outermost mesh point, where free-stream conditions are applied, is placed at $y=40$ in order to account for the fact that at high $E_{0}$ and low $\beta$ the perturbation at $x=1$ extends up to $y \approx 30$. The decision on where to truncate the Fourier series is taken by successively increasing $N$ and by comparing results with different spanwise resolutions. For low values of the initial energy, $N=5$ is found to be sufficient; as $E_{0}$ increases, up to $N=16$ (i.e. a total of 33 Fourier modes) has to be used to achieve grid-converged results.

\subsection{Different objective functions: recovering the linear results}

Results obtained by using two objective functions are shown in Fig. 1 for the optimal wavenumber, defined as the value of $\beta$ that maximizes $\mathcal{J}$ for a fixed $E_{0}$ (first two curves in the legend). The third curve of the legend is drawn for comparison purposes. A low value of the initial energy, $E_{0}=10^{-7}$, is used in order to ensure the linear regime and allow comparison with results available in the literature. In Fig. 1(a) the energy of the perturbation is represented as a function of the streamwise coordinate $x$. If the aim is the maximization of the energy at the final station, the maximum value is reached at $x=1$. On the contrary, if the target is the integral of the energy along the streamwise coordinate, the energy at the end is somewhat lower, but over a certain range of values of $x(x<0.8)$ it is larger than in the first case. It is remarkable, however, how close the two curves are to each other. This is contrary to what happens when an opposition control is applied [30], in which case the use of different objective functions produces very different behaviours. In Fig. 1(b) the optimal initial velocity profiles are reported. The initial perturbation that maximizes the final energy $E_{\text {out }}$ reproduces the one documented by Luchini [14] and Andersson et al. [16], and this validates the code in the linear regime.

The curve with $\mathcal{J}=E_{\text {mean }}$ and $\beta=0.45$ has been included in each of these two figures to try and discriminate whether the change in initial profile or the change in $\beta$, induced by the change in objective function, is mostly responsible for the result. It can be concluded that the choice of the objective function determines a sizeable change in both initial profile and $\beta$.

In Fig. 2 the results of the optimization procedure are shown at the outlet $x=1$. They are normalized with respect to $\sqrt{E_{\text {out }}}$ and correspond to the initial conditions reported in Fig. 1 . Since $v$ and $w$ are of order $R e^{-1 / 2}$ with respect to $u$, and are therefore much smaller, only the modulus of the $u$ component is reported. It is observed that the shape of the solution at the final station changes very little with the objective function, with the wavenumber and its ensuing optimal initial condition. This is in agreement with the early experimental observations by G.I. Taylor [35] and with the higher-singular-value analysis of Luchini [14] regarding the "near universality" of the output velocity profile in a linear context.

In the following, the functional $\mathcal{J}=E_{\text {mean }}$ is optimized in both the linear and nonlinear regimes, partly to explore this alternative to $\mathcal{J}=E_{\text {out }}$, the focus of all previous studies of optimal perturbations, and partly to provide a framework to flowcontrol results [31].

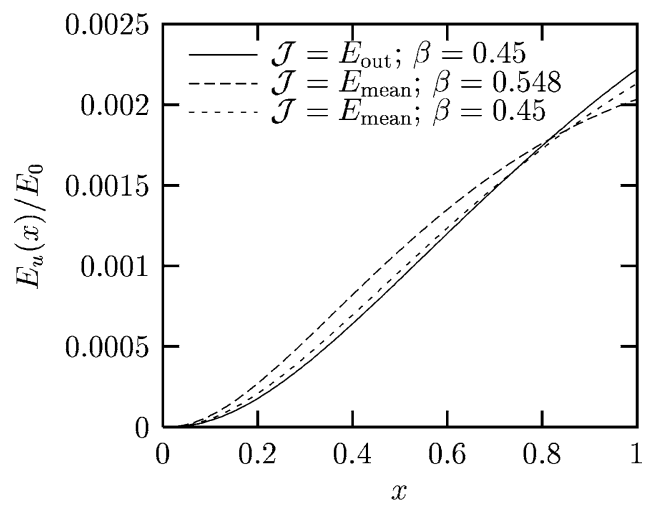

(a)

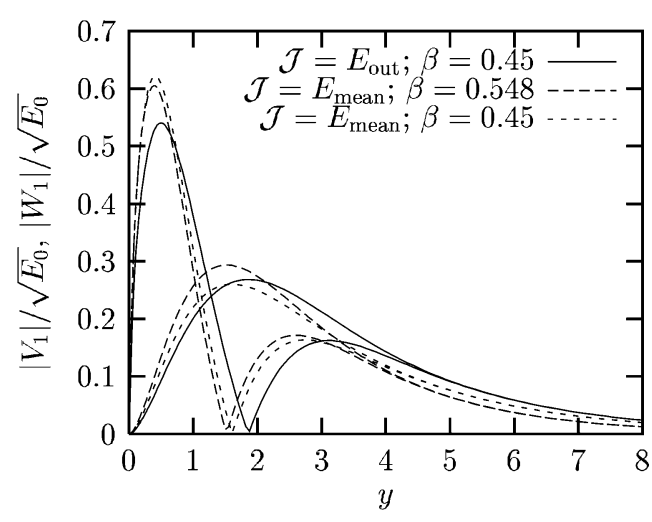

(b)

Fig. 1. Comparison between two different objective functions: (a) energy of the perturbation as a function of $x$, normalized with respect to the initial energy $E_{0}$. (b) Optimal perturbations normalized with respect to $\sqrt{E_{0}}$. The cases $\mathcal{J}=E_{\text {out }}$ at $\beta=0.45$ and $\mathcal{J}=E_{\text {mean }}$ at $\beta=0.548$, correspond to the optimal $\beta, \mathcal{J}=E_{\text {mean }}$ at $\beta=0.45$ is reported for comparison at the same wavenumber. $E_{0}=10^{-7}$, linear behaviour. 


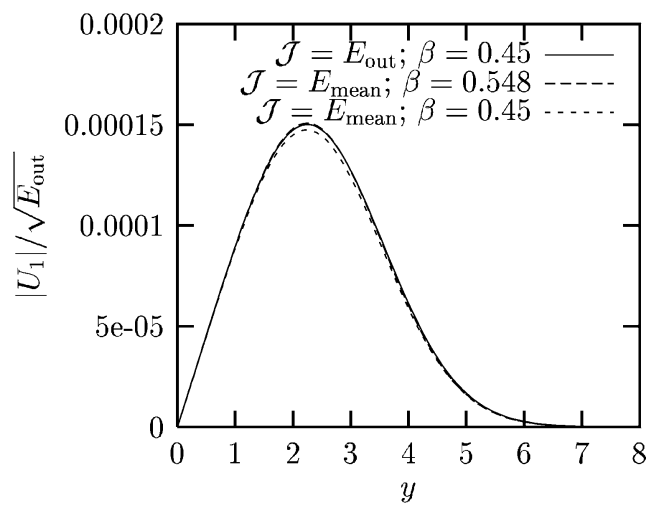

Fig. 2. Comparison between two different objective functions. Streamwise velocity component $U_{1}(1, y)$ at the final station $x=1$, normalized with respect to $\sqrt{E_{\text {out }}}$.

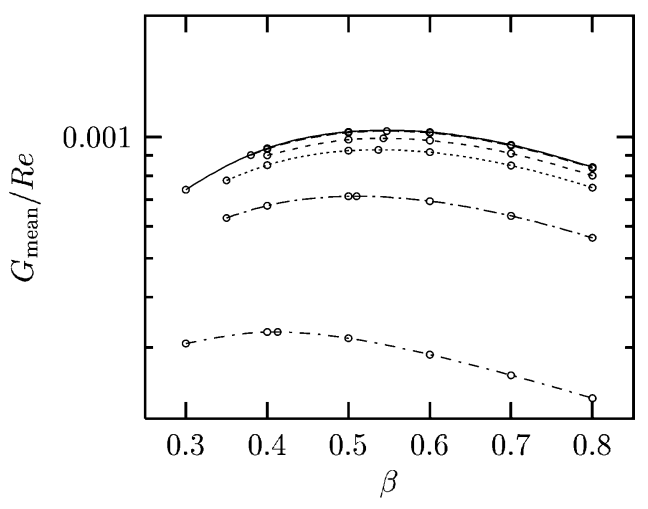

Fig. 3. Mean gain for different initial energy values $E_{0}$ and different wavenumbers. Linear case: $-; E_{0}=1$ :---; $E_{0}=10:--; \quad E_{0}=25: \cdots ; \quad E_{0}=100:-\cdots ;$ $E_{0}=500:-\cdot-$

\subsection{Nonlinear results}

In Fig. 3 the mean gain $G / R e$ is shown as a function of the wavenumber $\beta$ for different values of the initial energy $E_{0}$. The linear result (solid line) is obtained with a very low initial energy $\left(E_{0}=10^{-7}\right)$ and using only one spanwise mode. It can be noticed that increasing $E_{0}$ up to $E_{0}=1$ makes no visible difference, meaning that the nonlinear regime is not yet reached until $E_{0} \approx 10$. All curves present a maximum at a optimal wavenumber $\beta$ which depends on the value of $E_{0}$. With increasing $E_{0}$ the maximum gain shifts towards lower wavenumbers and this dependence is stronger and more evident for high $E_{0}$. In the linear regime, the optimum is found for $\beta=0.548$, which is more than $20 \%$ larger than the value found when $\mathcal{J}=E_{\text {out }}$. It must, however, be emphasized that these gain curves are all rather flat, i.e. there is no strong spanwise wavelength selectivity mechanism and the average wavenumber which is actually observed in experiments is strongly correlated to the spectrum of incoming disturbances (cf. [18]).

Incidentally we mention that the curves of Fig. 3, if continued to lower wavenumbers, exhibit ghost images due to the fact that the optimization algorithm converges on the same solution again, but for the second rather than the first harmonic.

According to Fig. 3, different comparisons can be set up to explore the parametric behaviour of optimal perturbations. For instance, the influence of the nonlinear interactions as $E_{0}$ grows can be investigated by fixing $\beta$ and increasing the initial energy or by comparing results at the optimal wavenumber. On the other hand, any possible dependence on the wavenumber can be studied by fixing $E_{0}$ and varying $\beta$. A further comparison between small and large values of $E_{0}$ can disclose differences due to nonlinear effects.

In Fig. 4(a) the perturbation energy $E_{u}$ of the streak (as defined in (7)), normalized with respect to the initial energy $E_{0}$, is reported as a function of $x$ for a fixed wavenumber $\beta=0.5$ and different $E_{0}$ 's up to 500. A linear amplification is clearly observed for $E_{0} \leqslant 1$ as all the curves collapse onto the linear result (solid line). For very high initial energy levels, on the contrary, the curves exhibit a saturation plateau (i.e. the flat region observable for $E_{0}=500$ at $0.7<x<1$ ). In Fig. 4(b) a section in the plane $z=0$ of the initial optimal perturbation $v_{0}(y, z)$ normalized with the square root of the initial energy $E_{0}$ is reported. Only the wall-normal velocity component is shown because $w_{0}(y, z)$ is simply proportional to the derivative of $v_{0}$ with respect to the wall-normal coordinate $\left(V_{n}\right.$ and $W_{n}$ are related by the continuity equation for each mode $\left.n\right)$. A departure from the linear behaviour can be observed even for $E_{0}=1$. This difference can be appreciated in physical space, while if only the first mode is compared, no significance discrepancy can be found until $E_{0}=10$. For increasing $E_{0}$, the maximum of the curve decreases and slightly shifts towards the wall. For $E_{0}=500$ the trend is interrupted and the maximum moves away from the wall, with a perturbation that decays slowly in $y$.

The difference between a low- $E_{0}$ and a high- $E_{0}$ case can be better appreciated by looking at the surface $v_{0}(y, z) / \sqrt{E_{0}}$ in the cross-stream plane for two different values of $E_{0}$. This is shown in Fig. 5; for $E_{0}=1$ there is only one peak, in the plane $z=0$; it is concentrated in a narrow region, and its value is higher than for $E_{0}=100$, for which the maximum spreads on a wider region of the plane $(y, z)$. This is a consequence of the nonlinear effects and if $E_{0}$ is further increased multiple maxima arise in the surface. In Fig. 6 the optimal disturbance velocity at $x=0$ is shown via vector plots. The figure confirms that the optimal perturbation is in the form of counter-rotating vortices and produces a lift-up/push-down effect. For $E_{0}=1$ the center of the vortices is located more or less in the center of the region occupied by the vortex itself. On the contrary, when $E_{0}$ is high, the vortex center moves away from the wall and towards the downwash region. 


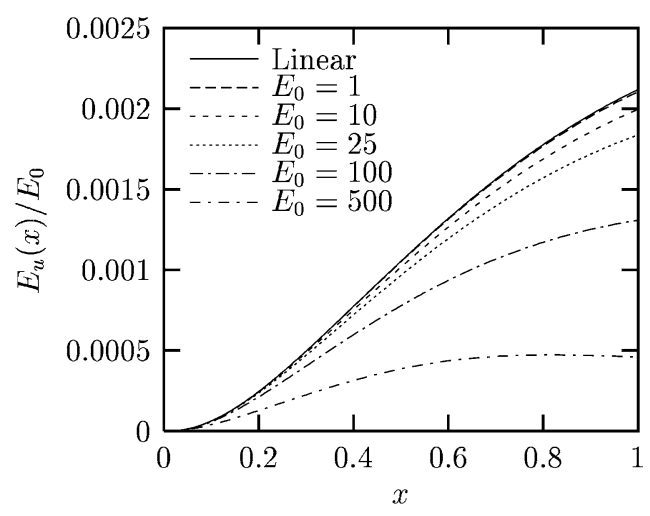

(a)

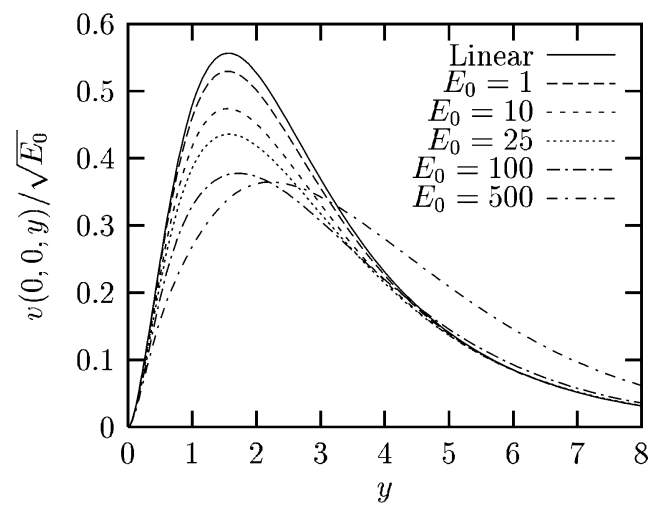

(b)

Fig. 4. Results at fixed wavenumber $\beta=0.5$ for increasing initial energy $E_{0}$. (a) Perturbation energy $E_{u}(x) / E_{0}$; (b) optimal perturbation $v_{0}(y, z) / \sqrt{E_{0}}$ in the plane $z=0$.

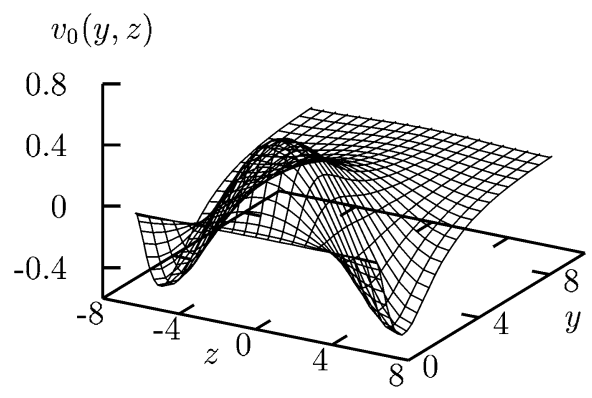

(a)

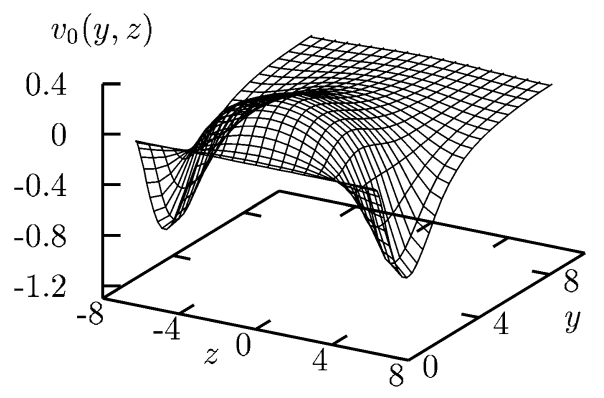

(b)

Fig. 5. Surfaces $v_{0}(y, z) / \sqrt{E_{0}}$ at fixed wavenumber $\beta=0.5$ for different $E_{0}$. (a) $E_{0}=1$; (b) $E_{0}=100$.

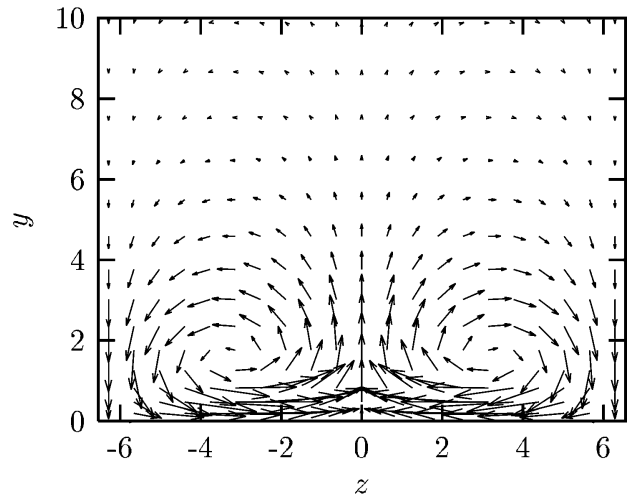

(a)

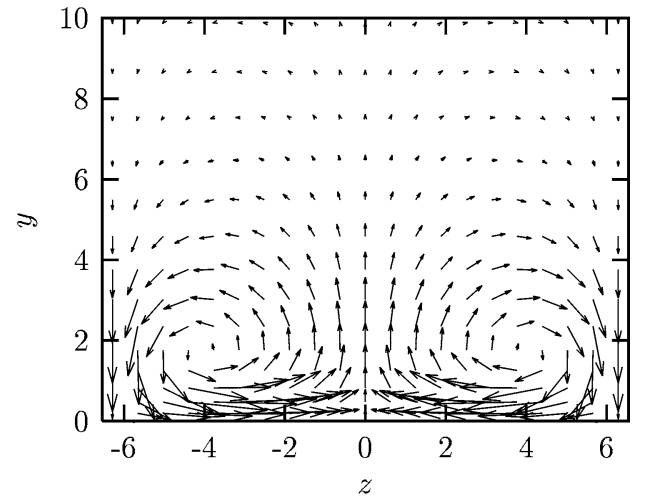

(b)

Fig. 6. Disturbance velocity vector plots in the cross-stream plane $(y, z)$ at $x=0$, for $\beta=0.5$. (a) $E_{0}=1$; (b) $E_{0}=100$.

In Fig. 7 contours of the $u$ velocity component are displayed at two streamwise locations, $x=0.5$ and $x=1$ (exit section) in order to emphasize the role of the streaks. The effect of nonlinearities increases with $x$, tending towards the typical "mushroom" shape characteristic of very high initial energies. The same kind of distortion was found by Andersson et al. [17].

Mode zero of the final $u$ - and $v$-profile, representing the mean flow contribution, is reported in Fig. 8, together with the Blasius solution. For $E_{0}>10$ the difference from Blasius' solution becomes noticeable, and for very high initial energies, for example $E_{0}=500$, the solution at $x=1$ inside the boundary layer is profoundly distorted and definitely different from Blasius', rendering the flow susceptible to secondary breakdown. This issue will be discussed in Section 4 . 


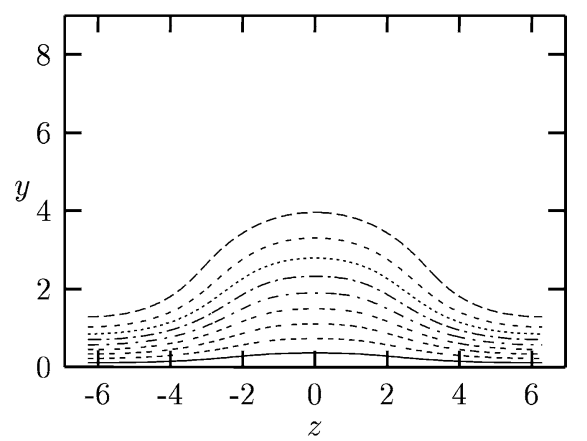

(a)

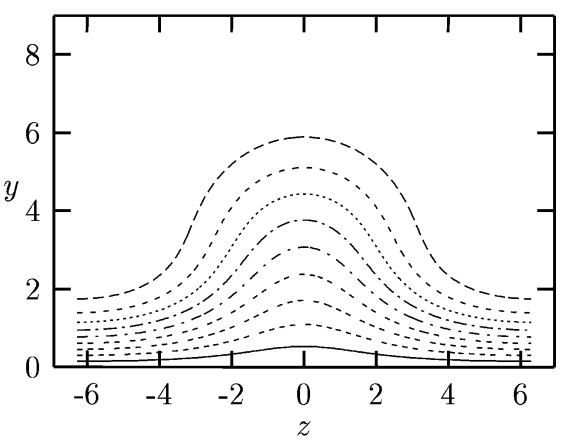

(b)

Fig. 7. $\beta=0.5, E_{0}=100$, contours of the streamwise velocity $u$ at: (a) $x=0.5$, (b) $x=1$.

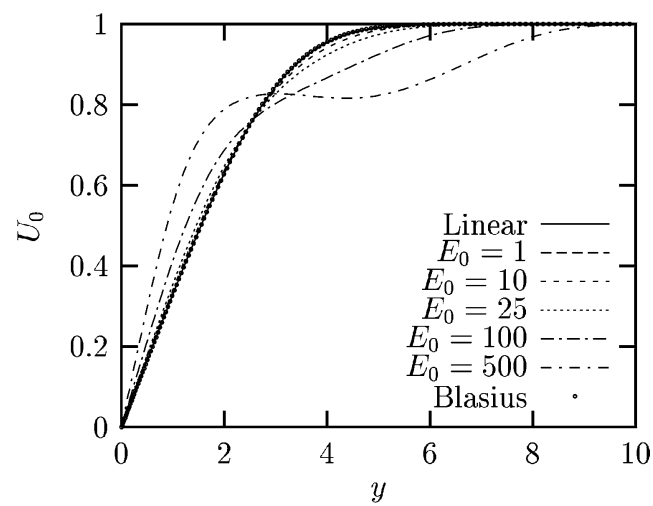

(a)

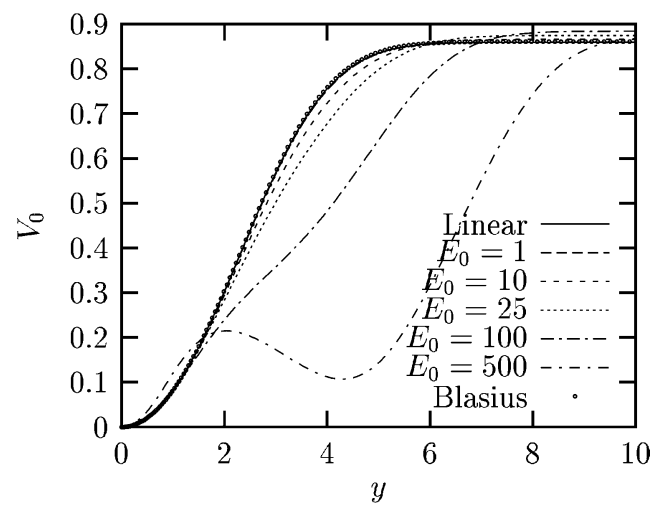

(b)

Fig. 8. Comparison at fixed wavenumber $\beta=0.5$. Mode zero at $x=1$ for increasing $E_{0}$.

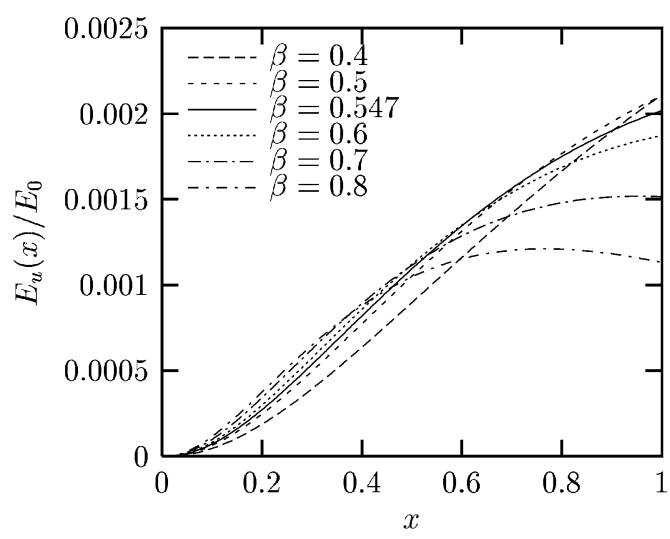

(a)

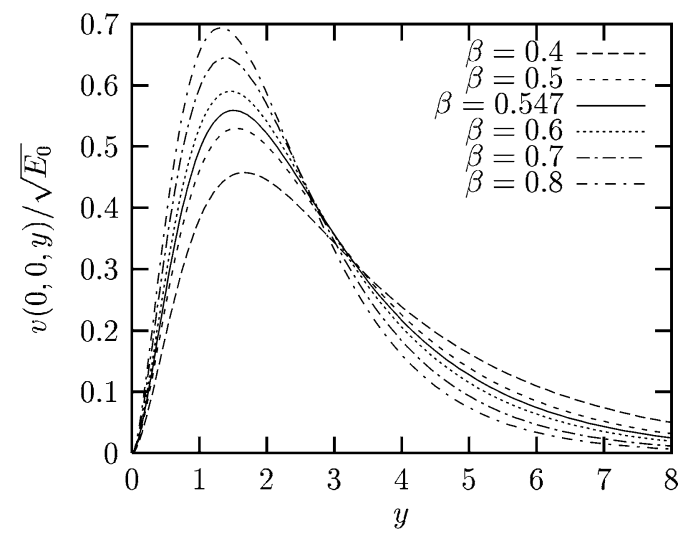

(b)

Fig. 9. Comparison at fixed initial energy $E_{0}=1$ for different values of the wavenumber $\beta$. (a) Perturbation energy $E_{u}(x) / E_{0}$; (b) optimal perturbation $v_{0}(y, z) / \sqrt{E_{0}}$ in the plane $z=0$. The optimal $\beta$ for this initial energy is plotted with solid lines.

Another manner of analyzing the results is to consider a fixed initial energy and varying wavenumber $\beta$. This is done in Fig. 9, where the energy $E_{u}$ as a function of $x$ and the optimal initial perturbation profile are reported for the case $E_{0}=1$. The energy plot, Fig. 9(a), indicates that the plateau observed for high $E_{0}$ at fixed $\beta=0.5$ (Fig. 4(a)) also occurs for very low initial energy, but at high wavenumbers. The straightforward conclusion is that the plateau is not directly related to the initial energy $E_{0}$ or to the action of nonlinearities, but seems to be a characteristic of wavenumbers higher than the optimal one. This is 


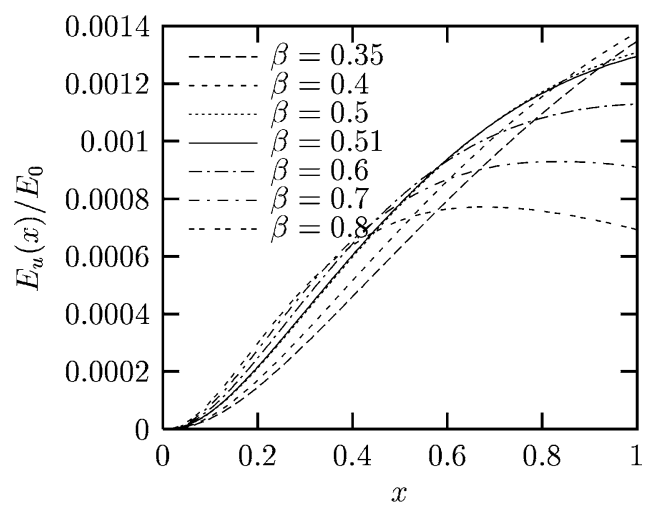

(a)

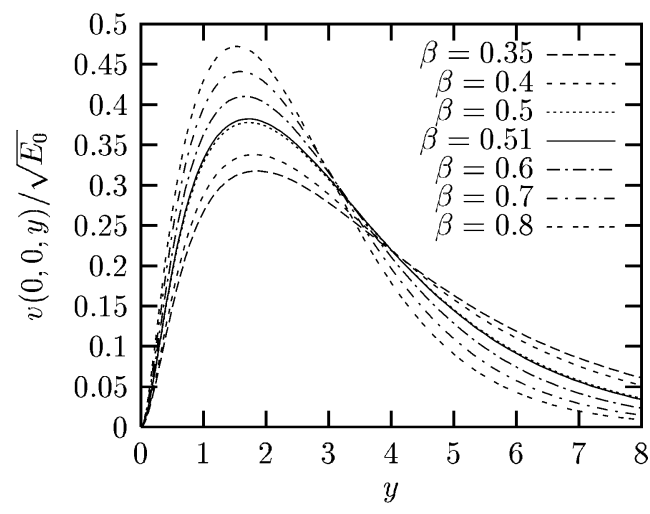

(b)

Fig. 10. Comparison at fixed initial energy $E_{0}=100$ for different values of the wavenumber $\beta$. (a) Perturbation energy $E_{u}(x) / E_{0}$; (b) optimal perturbation $v_{0}(y, z) / \sqrt{E_{0}}$ in the plane $z=0$. The optimal $\beta$ for this initial energy is plotted with solid lines.

confirmed by Fig. 10(a), where the energy behaviour is shown for the case $E_{0}=100$, displaying qualitatively similar features. The plateau is only present at large wavenumbers, thus supporting the conclusion that this feature depends primarily on the value of $\beta$. An interesting possible repercussion of this could be that small-wavelength streaks, being less energetic, are less easily destabilized by streamwise travelling wave disturbances. This statement must, however, be modulated by the realization that small-wavelength streaks present internal regions of high spanwise shear, known to cause and to be well correlated to sinuous travelling modes of instability [36,37].

Such a plateau can be also observed in the results of Andersson et al. [17], who solved the nonlinear boundary-layer equations for a fixed initial condition corresponding to the optimal linear perturbation at $\beta=0.45$. In that case, results are shown for different initial energies $E_{0}$ and the plateau is observed at high $E_{0}$ ( $\beta$ is fixed). According to Figs. 9(a) and 10(a), what found by Andersson et al. [17] can be ascribed to the fact that for those initial energies the wavenumber is higher than optimal.

An explanation of the dependence of the energy growth curve on $\beta$ might be found in the inviscid limit. For $v \rightarrow 0$ the forcing of the streaks is proportional to $\beta$ and therefore streaks with larger wavenumber will experience a larger initial growth. On the other hand, the damping effect of viscosity is proportional to $\beta^{2}$ so these perturbations also start to decay earlier. This behaviour, typical of large-wavenumber initial perturbations, is supported by the results displayed in Figs. 9(a) and 10(a).

From Figs. 9(b) and 10(b) one can notice that the shape of the optimal perturbation also changes with wavenumber, with the same trend for low- and high-initial-energy cases. The maximum shifts towards the wall and increases as $\beta$ increases. For low $\beta$ the profile goes to zero very slowly with $y$, as shown in Figs. 9(b) for $\beta=0.4$ and in 10 (b) for $\beta=0.3$.

The conclusion from Figs. 4-9 is that the wavenumber has a stronger influence on the shape of the optimal perturbation and its ensuing growth than the initial energy level, provided $E_{0}$ is not exceedingly large.

Finally, in Fig. 11 the solutions obtained for a variety of initial energy values $E_{0}$ are shown, at the optimal wavenumber (cf. Fig. 3 for an estimate of the optimal value of $\beta$ as $E_{0}$ varies). The first remark is that increasing the initial energy $E_{0}$ produces a variation in the energy growth but the change is much smoother than that observed at fixed $\beta$ or fixed $E_{0}$. The shape of the optimal perturbation at $x=0$ still shows a dependence on $E_{0}$, related to the fact that also $\beta$ changes.

\section{Links to streak breakdown}

It is important at this point to try and link the disturbance amplitude at $x=0$ to the possible breakdown of streaks, in order to provide approximate bounds for the initial energy below which destabilization of the streaks should not take place. It has been reported by Alfredsson (private communication, 1998) that "amplitudes of at least 20\%" are needed for an instability of the streaks to emerge. A similar threshold was detected by Bakchinov et al. [38] in their experiments in which streaks were generated by roughness elements distributed regularly along the span of the wall, and the instability was triggered by a vibrating ribbon upstream. In the inviscid linear stability analysis of Andersson et al. [17] the threshold amplitude for the occurrence of a sinuous (subharmonic) instability of the parallel streaky flow with $\beta=0.45$ was $26 \%$ of the free stream speed, whereas a larger value $(37 \%)$ was found for the amplification of a varicose mode. Results are not available for other spanwise wavelengths. 


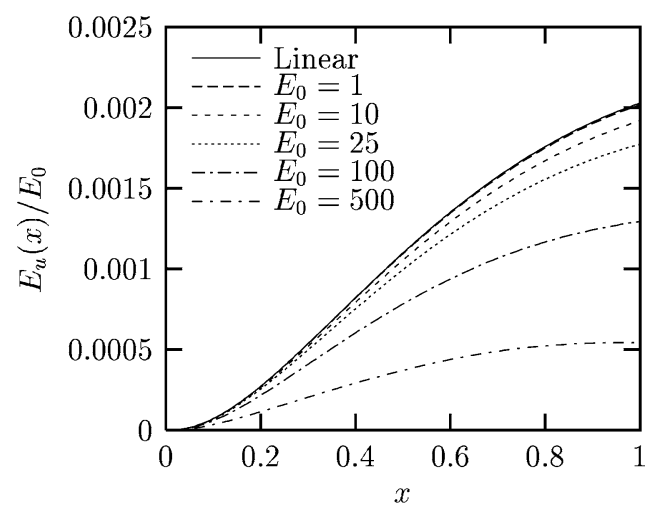

(a)

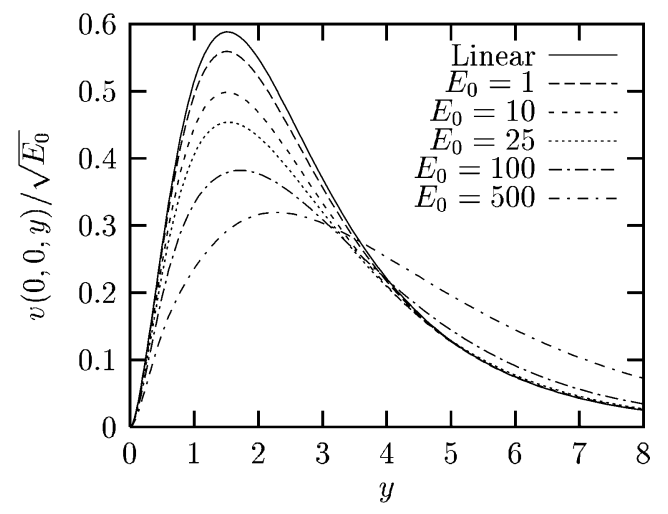

(b)

Fig. 11. Comparison at optimal wavenumber $\beta$ (defined as the wavenumber for which the curve of the gain reaches its maximum at each fixed initial energy $E_{0}$ ) for different values of $E_{0}$. (a) Perturbation energy $E_{u}(x) / E_{0} ;$ (b) optimal perturbation $v_{0}(y, z) / \sqrt{E_{0}}$ in the plane $z=0$.

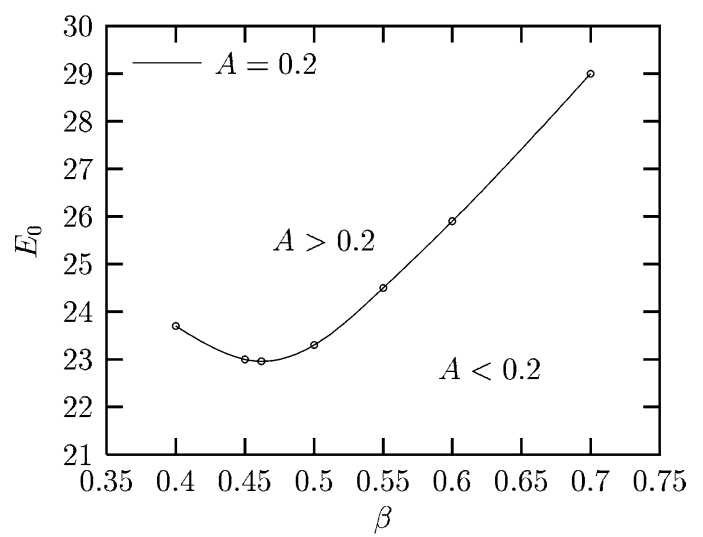

Fig. 12. Curve of initial perturbation energy $E_{0}$ as a function of $\beta$ for which $A=0.2$ somewhere in the domain.

To provide a conservative estimate of the threshold amplitude of the initial disturbance at $x=0$ that eventually leads to streaks that can break down, we take a "critical" amplitude of the nonlinear streaks $A$ equal to $20 \%$, with $A$ defined as in Andersson et al. [17]:

$$
A=\frac{1}{2}\left[\max _{y, z}\left(U-U_{B}\right)-\min _{y, z}\left(U-U_{B}\right)\right]
$$

where $U_{B}$ is the Blasius profile.

Since the results obtained so far for optimal nonlinear streaks give the functional form $A=A\left(x ; E_{0}, \beta\right)$, we can, for each given saturation value of $A$ and for each $\beta$, retrieve the corresponding initial energy level $E_{0}$. In a real physical situation the initial energy level capable of yielding the given value of $A$ at saturation will be larger, since leading edge conditions in a wind tunnel are not optimal (in the sense considered here), but this should not prevent us from searching for a conservative bound.

Fig. 12 reports the value of $E_{0}$ which yields a streak whose downstream amplitude reaches the value $A=0.2$ : the region below the curve is where a secondary instability of the streaks is not expected. It is noteworthy that the curve presents a minimum around $\beta=0.46$, which is very close to the linear optimal wavenumber $\beta=0.45$ found by Luchini [14] and Andersson et al. [16].

Within the limits of the arguments employed here one can argue that initial disturbance energies $E_{0}$ at $x=0$ lower than about 23 (with the present scaling employed for energy, it must be recalled that the value of 23 still needs to be divided by $R e$ ) do not meet the threshold required to trigger secondary instabilities and, as such, should not provoke streaks' breakdown nor the ensuing bypass transition. 


\section{Summary and conclusions}

This paper is devoted to the study of the steady, nonlinear, three-dimensional algebraically growing instability in the incompressible boundary layer past a flat plate. An adjoint-based optimization technique is used in order to determine those optimal perturbations at the leading edge that provide the maximum energy growth for a given initial energy.

The solution is decomposed in a finite number of Fourier modes along the spanwise direction $z$ and discretized using finite differences in $x$ and $y$. The velocity field can be viewed as the sum of a spanwise-uniform contribution due to mode zero, which represents the unperturbed base flow plus a mean flow correction, and a spanwise-varying contribution due to all the other modes. The energy of the latter contribution is taken as a measure of the growth of the algebraic instability.

The linear results by Luchini [14] and Andersson et al. [16] are reproduced by imposing a very low value of the initial energy (so that the interactions between modes can be neglected) and choosing the energy at the final station as the objective to be maximized. An extended study for a range of wavenumbers and initial energies is then performed with the aim of maximizing the integral of the energy over the whole domain. Results are compared at constant wavenumber, at constant initial energy and at the optimal wavenumber, defined as that value of $\beta$ for which the gain is a maximum.

If $E_{0}$ increases, the maximum of the curve representing $G_{\text {mean }} / R e$ as a function of $\beta$ shifts towards smaller wavenumbers. The input energy level below which nonlinear effects are negligible is determined and the mean velocity profile at the final station turns out to be indistinguishable from Blasius'; for higher initial energies nonlinear effects are clearly seen especially from the presence of strong distortions in the mean flow profiles at the end of the plate. The appearance of a plateau in the curve of the disturbance energy versus $x$ does not necessarily indicate nonlinear saturation; the same plateau takes place at very low $E_{0}$ and high $\beta$, and its presence is, hence, more of a characteristic of closely spaced streaks than of nonlinear effects.

At this stage it is still impossible to provide a complete account of bypass transition in boundary layers. In the present study we have aimed at identifying the leading-edge perturbations that maximize the disturbance kinetic energy of the flow, employing optimal-control theory; it is possible that a different choice of the cost functional would have produced a different response. In particular, it might be interesting to identify the optimal initial conditions capable of producing the most intense shear layers downstream, to try and link nonlinear optimization results to available experimental observations of burst and turbulent spots. This is obviously a formidable task, and the definition of an admissible objective function is far from simple. Also, in view of recent developments briefly reviewed in Section 1.3 it seems very promising for future work to focus on the search of finite amplitude travelling wave solutions in the boundary layer, by employing an unsteady model.

Finally, this work has extended the previous transient-growth theory to the fully nonlinear regime, providing the background for the optimal and robust control of nonlinear Klebanoff modes, an important task for laminar-flow-control technology.

\section{Acknowledgements}

The research by SZ at Politecnico di Milano was made possible by a Ph.D. grant awarded by CIRA, Centro Italiano di Ricerche Aerospaziali. The work was started in Toulouse, where SZ spent six months in the framework of a Marie Curie Training Site Fellowship awarded by the EU.

\section{Appendix A. Implementation and numerical solution of the direct problem}

In order to account for a general, not necessarily sinusoidal, spanwise dependence of the flow field, the solution is expanded in a Fourier series along the spanwise direction $z$. If

$$
f(x, y, z)=\sum_{n=-\infty}^{\infty} F_{n}(x, y) \mathrm{e}^{\mathrm{i} n \beta z}
$$

represents any one of the quantities $u, v, w, p$, the function $F_{n}(x, y)$ is the complex amplitude of the $n$th mode and depends on $x$ and $y$ only. Under the given expansion, each of the nonlinear terms in Eqs. (1) produces a double summation containing a convolution. Thus:

$$
\begin{aligned}
& \left(U_{n}\right)_{x}+\left(V_{n}\right)_{y}+\mathrm{i} n \beta W_{n}=0, \\
& \left(C_{n}^{U U}\right)_{x}+\left(C_{n}^{U V}\right)_{y}+\mathrm{i} n \beta C_{n}^{U W}-\left(U_{n}\right)_{y y}+n^{2} \beta^{2} U_{n}=0, \\
& \left(C_{n}^{U V}\right)_{x}+\left(C_{n}^{V V}\right)_{y}+\mathrm{i} n \beta C_{n}^{V W}-\left(V_{n}\right)_{y}+n^{2} \beta^{2} V_{n}+\left(P_{n}\right)_{y}=0, \\
& \left(C_{n}^{U W}\right)_{x}+\left(C_{n}^{V W}\right)_{y}+\mathrm{i} n \beta C_{n}^{W W}-\left(W_{n}\right)_{y y}+n^{2} \beta^{2} W_{n}+\mathrm{i} n \beta P_{n}=0,
\end{aligned}
$$


where the coefficient $C_{n}^{F G}(x, y)$ is defined as

$$
C_{n}^{F G}(x, y)=\sum_{k=\max (-N, n-N)}^{\min (N, n+N)} F_{k}(x, y) G_{n-k}(x, y)
$$

and the number of modes is restricted to $N$ for discretization purposes.

Since system (A.2) is parabolic, we choose a second-order backward finite-difference scheme. A first-order discretization has also been used for testing purposes. In addition, in account of the fact that the solution becomes singular at $x=0$, and thus a very high-density grid is required to preserve accuracy close to the leading edge, a non-uniform grid is employed. In the wall-normal direction, second-order central finite differences are used, over a non-uniform grid with points more tightly clustered near the wall. This choice of the grid and the fact that equations are written in conservative form allow us to compute the boundary layer solution without recurring to boundary layer coordinates.

Upon implicit discretization (A.2) becomes a system of nonlinear algebraic equations, which is handled by incomplete Newton iteration. A complete Newton linearization would involve a relatively large linear system, because the nonlinear coefficients $C_{n}^{F G}(x, y)$ couple every mode of each variable $(u, v, w)$ to every other one. The main drawback of this technique is that a very large system needs to be solved and thus a large amount of memory and computing time would be required. To overcome this difficulty we use an incomplete linearization in which modes with different $n$ are uncoupled, and a separate narrow-band algebraic system must be solved for each $n$. After each iteration the residue is computed and, if it is greater than a certain threshold, all the modes are computed again using the results from the previous iteration.

For this purpose, we linearize (A.2) and separate each mode $F_{n}$ in two contributions $F_{n}=\bar{F}_{n}+f_{n}$ where $\bar{F}_{n}$ is the current guess from the previous iteration and $f_{n}$ is a small unknown correction. On neglecting quadratic terms like $f_{k}(x, y) g_{n-k}(x, y)$ in the coefficient $C_{n}^{F G}(x, y)$, and pruning the summations

$$
\sum_{k=\max (-N, n-N)}^{\min (N, n+N)} \bar{F}_{k} g_{n-k} \text { and } \sum_{k=\max (-N, n-N)}^{\min (N, n+N)} f_{k} \bar{G}_{n-k}
$$

to the only terms $\bar{F}_{0} g_{n}$ and $f_{n} \bar{G}_{0}$ we obtain:

$$
C_{n}^{F G}(x, y) \approx \sum_{k=\max (-N, n-N)}^{\min (N, n+N)} \bar{F}_{k} \bar{G}_{n-k}+\bar{F}_{0} g_{n}+f_{n} \bar{G}_{0}=C_{n}^{\overline{F G}}+\bar{F}_{0} g_{n}+f_{n} \bar{G}_{0} .
$$

After these modifications, the original nonlinear coupling coefficient $C_{n}^{F G}(x, y)$ has been reduced to a linear form that couples mode $n$ and mode zero only. It should be noted that, since at convergence all $f_{n}$ 's are zero, the converged solution is not altered at all; only the rate of convergence is, but on the other hand each iteration is now much faster. The system of equations thus reads:

$$
K_{0} \mathbf{A} \mathbf{f}_{n}+\mathbf{B}_{n} \mathbf{f}_{n}=-\mathbf{r}_{n}
$$

where $K_{0}$ accounts for the $x$-derivative (first or second order); the vector of unknowns is

$$
\mathbf{f}_{n}=\left(\begin{array}{c}
u_{n} \\
v_{n} \\
w_{n} \\
p_{n}
\end{array}\right)
$$

and

$$
\mathbf{A}=\left(\begin{array}{cccc}
1 & 0 & 0 & 0 \\
2 \bar{U}_{0} & 0 & 0 & 0 \\
\bar{V}_{0} & \bar{U}_{0} & 0 & 0 \\
\bar{W}_{0} & 0 & \bar{U}_{0} & 0
\end{array}\right), \quad \mathbf{B}_{n}=\left(\begin{array}{cccc}
0 & (\cdot)_{y} & \mathrm{i} n \beta & 0 \\
B_{21} & \left(\bar{U}_{0} \cdot\right)_{y} & \mathrm{i} n \beta \bar{U}_{0} & 0 \\
0 & B_{32} & \mathrm{i} n \beta \bar{V}_{0} & (\cdot)_{y} \\
0 & \left(\bar{W}_{0} \cdot\right)_{y} & B_{43} & \text { in } \beta
\end{array}\right),
$$

with

$$
\begin{aligned}
& B_{21}=\left(\bar{V}_{0} \cdot\right)_{y}+\mathrm{i} n \beta \bar{W}_{0}-(\cdot)_{y y}+n^{2} \beta^{2}, \\
& B_{32}=\left(2 \bar{V}_{0} \cdot\right)_{y}+\mathrm{i} n \beta \bar{W}_{0}-(\cdot)_{y y}+n^{2} \beta^{2}, \\
& B_{43}=\left(\bar{V}_{0} \cdot\right)_{y}+2 \mathrm{i} n \beta \bar{W}_{0}-(\cdot)_{y y}+n^{2} \beta^{2} .
\end{aligned}
$$


The residue $\mathbf{r}_{n}$, which must be driven to zero by the iteration, can be rewritten as $\mathbf{r}_{n}=\mathbf{r}_{0}+\left(\mathbf{r}_{1}\right)_{x}$, where $\mathbf{r}_{1}$ contains the terms to be differentiated in $x$ and $\mathbf{r}_{0}$ all the other terms:

$$
\mathbf{r}_{0}=\left(\begin{array}{c}
\left(\bar{V}_{n}\right)_{y}+\mathrm{i} n \beta \bar{W}_{n} \\
\left(C_{n}^{\bar{U}} \bar{V}\right)_{y}+\mathrm{i} n \beta C_{n}^{\bar{U}} \bar{W}-\left(\bar{U}_{n}\right)_{y y}+n^{2} \beta^{2} \bar{U}_{n} \\
\left(C_{n}^{\bar{V}} \bar{V}\right)_{y}+\mathrm{i} n \beta C_{n}^{\bar{V} \bar{W}}-\left(\bar{V}_{n}\right)_{y y}+n^{2} \beta^{2} \bar{V}_{n}+\left(\bar{P}_{n}\right)_{y} \\
\left(C_{n}^{\bar{V}} \bar{W}\right)_{y}+\mathrm{i} n \beta C_{n}^{\bar{W}} \bar{W}-\left(\bar{W}_{n}\right)_{y y}+n^{2} \beta^{2} \bar{W}_{n}+\mathrm{i} n \beta \bar{P}_{n}
\end{array}\right), \quad \mathbf{r}_{1}=\left(\begin{array}{c}
\bar{U}_{n} \\
C_{n}^{\bar{U} \bar{U}} \\
C_{n}^{\bar{U} \bar{V}} \\
C_{n}^{\bar{U}} \bar{W}
\end{array}\right) .
$$

The numerical solution of the direct problem is obtained by marching in the $x$-direction, from $x=0$ to $x=1$, while at each step driving the iterative solution of the implicit difference equations to convergence.

\section{References}

[1] B. Hof, A. Juel, T. Mullin, Scaling of the turbulence transition threshold in a pipe, Phys. Rev. Lett. 91 (24) (2003) 244502.

[2] T. Ellingsen, E. Palm, Stability of linear flow, Phys. Fluids 18 (1975) 487-488.

[3] M.T. Landahl, A note on an algebraic instability of inviscid parallel shear flow, J. Fluid Mech. 98 (1980) 243-251.

[4] H.K. Moffatt, The interaction of turbulence with strong wind shear, in: A.M. Yaglom, V.I. Tatarski (Eds.), Proc. URSI-IUGG International Colloquium on 'Atmospheric Turbulence and Radio Wave Propagation', Moscow, June 1965, Nauka, Moscow, 1967, pp. 139-154.

[5] M.V. Morkovin, Bypass transition to turbulence and research desiderata, NASA Conf. Pub. 2386, 1984.

[6] M.V. Morkovin, E. Reshotko, Dialogue on progress and issues in stability and transition research, in: D. Arnal, R. Michel (Eds.), LaminarTurbulent Transition, Springer, 1990, pp. 3-29.

[7] P. Luchini, Reynolds-number-independent instability of the boundary layer over a flat surface, J. Fluid Mech. 327 (1996) 101-115.

[8] B. Farrell, Optimal excitation of perturbations in viscous shear flow, Phys. Fluids 31 (1988) 2093-2102.

[9] L. Boberg, U. Brosa, Onset of turbulence in a pipe, Z. Natursforschung 43a (1988) 697-726.

[10] K.M. Butler, B. Farrell, Three-dimensional optimal perturbations in viscous shear flow, Phys. Fluids A 4 (1992) 1637-1650.

[11] P. Corbett, A. Bottaro, Optimal perturbations for boundary layers subject to streamwise pressure gradient, Phys. Fluids 12 (2000) 120-130.

[12] P. Corbett, A. Bottaro, Optimal linear growth in swept boundary layers, J. Fluid Mech. 435 (2001) 1-23.

[13] P. Luchini, Effects on a flat-plate boundary layer of free-stream longitudinal vortices: optimal perturbations, in: EUROMECH 3rd European Fluid Mechanics Conference, Göttingen, 15-18 September 1997, DLR, Göttingen, 1997, p. 219.

[14] P. Luchini, Reynolds-number-independent instability of the boundary layer over a flat surface: optimal perturbations, J. Fluid Mech. 404 (2000) 289-309.

[15] P. Andersson, M. Berggren, D.S. Henningson, Optimal disturbances in boundary layers, in: J.T. Borggaard, J. Burns, E. Cliff, S. Schreck (Eds.), AFOSR Workshop on Optimal Design and Control, Arlington, VA, USA, 30 September-3 October 1997, Birkhäuser, Boston, 1998.

[16] P. Andersson, M. Berggren, D.S. Henningson, Optimal disturbances \& bypass transition in boundary layers, Phys. Fluids 11 (1999) 134150.

[17] P. Andersson, L. Brandt, A. Bottaro, D. Henningson, On the breakdown of boundary layer streaks, J. Fluid Mech. 428 (2001) 29-60.

[18] M. Matsubara, P.H. Alfredsson, Disturbance growth in boundary layers subjected to free-stream turbulence, J. Fluid Mech. 430 (2001) $149-168$.

[19] D.W. Wundrow, M.E. Goldstein, Effect on a laminar boundary layer of small-amplitude streamwise vorticity in the upstream flow, J. Fluid Mech. 426 (2001) 229-262.

[20] R.G. Jacobs, P.A. Durbin, Simulations of bypass transition, J. Fluid Mech. 428 (2001) 185-212.

[21] F. Waleffe, Three-dimensional coherent states in plane shear flows, Phys. Rev. Lett. 81 (1998) 4140.

[22] F. Waleffe, Exact coherent structures in channel flow, J. Fluid Mech. 435 (2001) 93-102.

[23] F. Waleffe, Homotopy of exact coherent structures in plane shear flows, Phys. Fluids 15 (2003) 1517-1534.

[24] B. Hof, C.W.H. van Doorne, J. Westerweel, F.T.M. Nieuwstadt, H. Faisst, B. Eckhardt, H. Wedin, R.R. Kerswell, F. Waleffe, Experimental observation of nonlinear traveling waves in turbulent pipe flow, Science 305 (24) (2004) 1594-1598.

[25] H. Faisst, B. Eckhardt, Travelling waves in pipe flow, Phys. Rev. Lett. 91 (2003) 224502.

[26] H. Wedin, R.R. Kerswell, Exact coherent structures in pipe flow: travelling waves solutions, J. Fluid Mech. 508 (2004) 333-371.

[27] S.C. Plasting, R.R. Kerswell, A friction factor bound for transitional pipe flow, Phys. Fluids 17 (2005) 011706.

[28] L. Ting, On the initial conditions for boundary layer equations, J. Math. Phys. 44 (1965) 353.

[29] P. Luchini, A. Bottaro, Görtler vortices: a backward-in-time approach to the receptivity problem, J. Fluid Mech. 363 (1998) 1-23.

[30] P. Cathalifaud, P. Luchini, Algebraic growth in boundary layers: optimal control by blowing and suction at the wall, Eur. J. Mech. B Fluids 19 (2000) 469-490.

[31] S. Zuccher, P. Luchini, A. Bottaro, Algebraic growth in a Blasius boundary layer: optimal and robust control by mean suction in the nonlinear regime, J. Fluid Mech. 513 (2004) 135-160.

[32] P. Luchini, A. Bottaro, Linear stability and receptivity analyses of the Stokes layer produced by an impulsively started plate, Phys. Fluids 13 (2001) 1668-1678. 
[33] M. Gunzburger, Adjoint equation-based methods for control problems in incompressible, viscous flows, Flow, Turb. \& Comb. 65 (2000) 249-272.

[34] A.M. Moore, B.F. Farrell, Rapid perturbation growth on spatially and temporally varying oceanic flows determined using an adjoint method: Application to the Gulf Stream, J. Phys. Oceanogr. 23 (1993) 1682-1702.

[35] G.I. Taylor, Some recent developments in the study of the turbulence, in: J.P. den Hartog, H. Peters (Eds.), Proc. Fifth Intl. Congress for Applied Mechanics, Cambridge, MA, USA, September 12-16, 1938, Wiley, 1939, pp. 294-310.

[36] J.D. Swearingen, R.F. Blackwelder, The growth and breakdown of streamwise vortices in the presence of a wall, J. Fluid Mech. 182 (1987) 255-290.

[37] A. Bottaro, B.G.K. Klingmann, On the linear breakdown of Görtler vortices, Eur. J. Mech. B Fluids 15 (1996) 301-330.

[38] A.A. Bakchinov, G.R. Grek, B.G.B. Klingmann, V.V. Kozlov, Transition experiments in a boundary layer with embedded streamwise vortices, Phys. Fluids 7 (1995) 820-832. 